\title{
Science with the World Space Observatory - Ultraviolet
}

\author{
Ana I. Gómez de Castro ${ }^{1}$, Isabella Pagano ${ }^{2}$, Mikhail Sachkov ${ }^{3}$, Alain \\ Lecavelier $^{4}$, Giampaolo Piotto ${ }^{5}$, Rosa González ${ }^{6}$, and Boris Shustov ${ }^{3}$ \\ 1 Fac. de CC. Matemáticas, Universidad Complutense de Madrid, Plaza de \\ Ciencias 3, E-28040 Madrid, Spain aig@mat.ucm.es \\ 2 INAF - Osservatorio Astrofisico di Catania, v. S. Sofia 78, 95123 Catania, Italy \\ isabella.pagano@oact.inaf.it \\ 3 Institute of Astronomy of the Russian Academy of Science, 48 Pyatnitskaya St., \\ 119017 Moscow, Russia sachkov@inasan.ru \\ 4 Institute d'Astrophysique de Paris, 98bis, bd Arago - 75014 Paris FRANCE \\ lecaveli@iap.fr \\ 5 Dipartimento di Astronomia, Università di Padova, Vicolo dell'Osservatorio, 3 \\ 35122 Padova, Italy giampaolo.piotto@unipd.it \\ 6 Instituto de Astrofisica de Andalucia, Camino Bajo de Huetor 50, E-18008 \\ Granada, Spain rosa@iaa.es
}

\begin{abstract}
Summary. The World Space Observatory-Ultraviolet (WSO-UV) will provide access to the UV range during the next decade. The instrumentation on board will allow to carry out high resolution imaging, high sensitivity imaging, high resolution $(\mathrm{R} \sim 55000)$ spectroscopy and low resolution $(\mathrm{R} \sim 2500)$ long slit spectroscopy. In this contribution, we briefly outline some of the key science issues that WSO-UV will address during its lifetime. Among them, of special interest are: the study of galaxy formation and the intergalactic medium; the astronomical engines; the Milky Way formation and evolution, and the formation of the Solar System and the atmospheres of extrasolar planets.
\end{abstract}

\section{Introduction}

Launched at the beginning of the next decade, the World Space Observatory - Ultraviolet (WSO-UV) will fly for 5 years with a probable extension of other 5 years. The observatory will be in a circular geosynchronous orbit with $35800 \mathrm{~km}$ radius, with 51.8 degrees inclination; Earth occultation will be small and the orbital period will allow to time-track targets and to have rapid access to targets of opportunity.

The WSO-UV will be equipped with multipurpose instrumentation to carry out: 
- High resolution spectroscopy ( $\mathrm{R} \sim 55000)$ of point sources in the range 102$320 \mathrm{~nm}$ by means of two high resolution echelle set-ups - HIgh Resolution Double Echelle Spectrograph (HIRDES). The sensitivity of this instrument is 10 times better that of the Space Telescope Imaging Spectrograph (STIS) on a similar configuration.

- Long-slit low resolution ( $\mathrm{R} \sim 1500-2500)$ spectroscopy in the $102-320 \mathrm{~nm}$ range.

- High resolution (0.03 $\operatorname{arcsec}$ pixel $\left.^{-1}\right)$ imaging in the $150-280 \mathrm{~nm}$ range with a field of view of $1 \times 1 \mathrm{arcmin}^{2}$.

- High resolution (0.07 arcsec pixel $\left.{ }^{-1}\right)$ imaging in the $200-700 \mathrm{~nm}$ range with a field of view of $4.7 \times 4.7 \mathrm{arcmin}^{2}$. This camera will be in average a factor of 3 more effective sensitive than the Advanced Camera System (ACS) and the Wide Field Camera 3 on the Hubble Space Telescope (HST).

- A large field $\left(6.6 \times 6.6 \operatorname{arcmin}^{2}\right)$ far ultraviolet $(115-190 \mathrm{~nm})$ camera with spatial resolution 0.2 arcsec pixel ${ }^{-1}$. This will allow Lyman- $\alpha$, C IV or molecular hydrogen mapping in extended, faint, astrophysical targets.

General information on the WSO-UV mission are given by Sachkov et al. (this proceedings), while detailed information on the focal plane instruments can be found in Pagano et al. (this proceedings).

The Ultraviolet (UV) is a fundamental energy domain since it grants high sensitivity access to the study of both atomic plasmas at temperatures in the 3,000-300,000 K range and molecular plasmas illuminated by UV radiation since the electronic transitions of the most abundant molecules in the Universe $\left(\mathrm{H}_{2}, \mathrm{CO}, \mathrm{OH}, \mathrm{CS}, \mathrm{CO}_{2}^{+}, \mathrm{C}_{2} \ldots\right)$ are in this range; the $\mathrm{UV}$ radiation field itself is a powerful astrochemical and photoionizing agent. For this reason, most of the areas of astrophysics will benefit from the availability of a world-class observatory as WSO-UV during the next decade. A detailed accounting of the needs for UV instrumentation in the many fields of astrophysical research can be found in the special issue entitled Fundamental problems in astrophysics: requirements for future UV observatories (Gómez de Castro \& Wamsteker, eds., 2006) that summarizes the thoughts and work of the Network of UV Astronomy $\left(\mathrm{NUVA}^{7}\right)$ and the UV community at-large.

The WSO-UV will work as a Space Observatory with a core program, guaranteed time for the project partners and time open to excellent scientific projects from the world-wide community. In this contribution we outline some of the key issues in the WSO-UV Observatory program:

1. Galaxy formation: determination of the diffuse baryonic content in the Universe and its chemical evolution.

2. The physics of accretion and outflow: the astronomical engines.

3. The Milky Way formation and evolution

4. Extrasolar planetary atmospheres and astrochemistry in the presence of strong UV radiation fields.

\footnotetext{
${ }^{7}$ http:www.ucm.es/info/nuva/
} 


\section{Determination of the diffuse baryonic content in the Universe and its chemical evolution}

The composition of the Universe (baryonic matter, dark matter and dark energy densities) is derived from observations related to the first 3 Gyr the Universe (Cosmic Background, high z quasars and ultraluminous galaxies at $z>3$ ). To understand the physical transition from this early epoch to our current Universe (14 Gyr) it is necessary to get information on the recycling of the gas (InterGalactic Medium, IGM) and the evolution of the stellar population and the radiation field over the redshift interval $0<z<2$ that spans a $80 \%$ of the lifetime of the Universe. Though theoretical modelling on structure and galaxy formation is progressing rapidly there are no data to contrast with since the IGM is poorly studied. Fundamental parameters such as the galaxy formation efficiency and its variation across the Universe cannot be measured since data about the gas component are, at the very least, scarce. Fundamental questions such as, which physical forces trigger galaxy formation or what controls the galaxy mass function and the lower limits to galactic masses cannot be addressed without a good knowledge of the IGM properties.

UV spectroscopy and imaging are the most sensitive technique to obtain direct information in the $z<2$ Universe on:

\subsection{The baryonic content of the Warm-Hot IGM}

Independent of the different models for the early Universe the major baryonic component of the Universe at $z<3$ must be associated with the IGM. The Warm-Hot component of the IGM (WHIM), at temperatures $T=10^{5}-10^{7} \mathrm{~K}$, most likely accounts for $\mathrm{a} \sim 30 \%$ of the baryons to the cosmological mass density in the local Universe (see e.g. the cosmological simulations by Cen \& Ostriker 1999). Broad HI-Ly $\alpha$ absorption is one of the most promissing techniques to map the distribution of the WHIM.

\subsection{The Damped Ly- $\alpha$ (DLA) systems}

DLA systems trace column densities about $10^{20} \mathrm{~cm}^{-3}$, e.g. dense clumps of gas likely associated with galaxy formation sites. Numerical simulations show that these clumps arise naturally in the hierarchical theory of galaxy formation (Katz et al 1996) and thus become the best tracers to the characteristic scales of structure formation between the early epochs and the local Universe. DLAs trace the location of the gravitational potential wells of all the matter, henceforth representing another tracer of dark matter.

\subsection{He II reionization}

From theoretical modeling of the IGM we know that after the HI reionization, the IGM cools by expansion, is reheated by the delayed HeII reionization 
at $\mathrm{z}=3$, and continues to cool with decreasing redshift. Observations of the HeII $\lambda 304 \AA$ forest over the redshift range $2.1<z<2.9$ will test this model in the most direct way (Reimers et al 2005).

\subsection{The role of starbursts in the evolution of the IGM}

Starbursts are systems with very high star formation rate per unit area. They are the preferred place where massive stars form; the main source of thermal and mechanical heating in the interstellar medium, and the factory where the heavy elements form. Thus, starbursts play an important role in the origin and evolution of galaxies. The similarities between the physical properties of local starbursts and high- $z$ star-forming galaxies, highlight the cosmological relevance of starbursts. Moreover, nearby starbursts are laboratories where to study violent star formation processes and their interaction with the interstellar and intergalactic media, in detail and deeply. Outflows in cold, warm and coronal phases leave their imprints on the UV interstellar lines. Outflows of a few hundred $\mathrm{km} \mathrm{s}^{-1}$ are ubiquitous phenomena in starbursts. These metalrich outflows and the ionizing radiation can travel to the halo of galaxies and reach the intergalactic medium. Observations with the WSO-UV will allow to study the stellar content and the high mass IMF of the starburst and to determine the chemical composition of the associated galactic winds.

\subsection{Galaxies formation}

How the present-day Hubble sequence of galaxies was assembled, as traced by the buildup of their mass, gas, stars, metals, and magnetic fields? Understanding the formation and evolution of galaxies is driving most of the current efforts in observational cosmology. Galaxies are thought to form inside the dark matter (DM) halos produced by gravitational instability from the seed initial fluctuations. The evolution of the DM component is successfully predicted from first principles, because it evolves under the sole action of gravity. However, the predictive power of theory drops dramatically when it comes to the luminous, baryonic component of the universe. Complex physical processes come into play, and it is no surprise that the attempts at modelling galaxies have so far attained limited success. A number of different investigations with WSO-UV imagers can provide fundamental inputs to the problem of galaxy formation and evolution. For instance, high resolution and deep images in wide bands at $150 \mathrm{~nm}, 220 \mathrm{~nm}$, and 300nm imaging of GOODS/UDF Deep Fields (Dickinson et al. 2003), allow to:

1. Search for Lyman Break Galaxies at relatively low z. While the search for LBG galaxies is a very well established technique at $\mathrm{z} \geq 2-3$, the interval around $\mathrm{z} \sim 1-1.5$ is poorly explored due to the lack of deep and good angular resolution observations. 
2. Study of the UV luminosity function: the UV (rest frame $150 \mathrm{~nm}$ ) luminosity function is currently poorly constrained at the faint end. The 300 $\mathrm{nm}$ observations will fill the gap in particular at $\mathrm{z} \sim 1-1.5$, where the global cosmic star formation rate stars to decline.

\section{Astronomical engines}

Astronomical engines (stars, black holes, etc...) can accelerate large masses to velocities close to the speed of light or generate sudden ejections of mass as observed in Supernova explosions. They are also able to produce significantly milder winds, as seen in the Sun, or to eject gas shells induced by pressure pulsations in the stellar atmosphere. All of these phenomena transform energy of various forms (gravitational, thermal, radiative, magnetic) into mechanical energy to produce outflows in conditions very different to those tested in Earth laboratories. The least conventional engines are those generating highly collimated bipolar outflows and jets. These are thought to be driven by a combination of gravitational energy, differential rotation and magnetic fields. They are among the most exciting objects in nature; however, their underlying physics is poorly known. This physical regime affects all of the many scales of Astrophysics; it determines the luminosity of the Active Galactic Nucleii and the re-ionization of the Universe at $z \simeq 3$. It also determines the properties of planetary systems, which are just angular momentum reservoirs left over when the engine is turned off in pre-main sequence stars.

WSO-UV will provide key inputs to answer the basic open questions concerning this physics:

1. What controls the efficiency of accreting objects as gravitational engines?, is the magnetic field needed to guarantee that outflows are fast?, what are the relevant timescales for mass ejection?

2. How does the accretion flow proceed from the disk to the source of the gravitational field in the presence of moderate magnetic fields?, which fraction of the gravitational energy lost in this process is deposited on the stellar surface?, which fraction is lost in amplification/dissipation of magnetic flux?

3. Which is the role played by radiation pressure in this whole process?

4. What role do disk instabilities play in the whole accretion/outflow process?, which are the key mechanisms driving these instabilities?

Some few examples are:

1. High resolution UV spectroscopy will allow to determine the structure of the accretion flow on magnetic cataclismic variables and on $\mathrm{T}$ Tauri stars and to measure the physical conditions and clumpyness of the outflows. It will also allow to study the source of energy that powers the extended dense $\left(\geq 10^{10} \mathrm{~cm}^{-3}\right)$ and hot $\left(\mathrm{T}_{e} \geq 60,000 \mathrm{~K}\right)$ envelopes that have been 
detected around T Tauri stars (Gómez de Castro \& Verdugo, 2003). The luminosities of these envelopes are about $0.2 \mathrm{~L}_{\odot}$.

2. Low resolution spectroscopy will allow to measure the general physical conditions and metalicities of the Broad Lines Emission Region in Active Galactic Nucleii. Reverberation mapping will allow to study the kinematics and the mass of the central supermassive black holes. Also the atmospheres of the hot accretion disks in cataclysmic variable stars will be studied and the role of disk instabilities in triggering the outbursts.

3. The high sensitivity FUV camera will allow to detect hot jets through their Ly- $\alpha$ and to resolve the thermal structure of the jets and the regions shocked by them. It will also allow to survey star forming regions to detect planetary-mass objects in regions like $\sigma$-Orionis and to study the magnetic activity and accretion processes in these free-floating planetary-like objects which are at the low mass end of the molecular clouds fragmentation scales.

\section{How did our Galaxy form?}

What is the detailed history of the formation and evolution of our own Galaxy, and what lessons it hold for the formation and evolution of galaxies generally. Globular clusters have traditionally been considered as good tracers of the process that led to the formation of their host galaxy. Absolute ages set a constraint on the epoch of the galaxy formation, with important cosmological consequences. Relative ages provide detailed information on the formation process of the host galaxy. For the absolute age determination, accurate distances, metallicity, and reddening are mandatory. As for the distance, while waiting for GAIA results, there is a simple, geometric method to get accurate distances of GCs: the comparison of the dispersion of internal proper motions, an angular quantity, with that of radial velocities, a linear quantity gives a distance. Radial velocities for thousands of stars in GCs, with an accuracy of a few hundred $\mathrm{km} / \mathrm{s}$ are now easily attainable with multifiber facilities at the 8-10m class telescopes. As for the proper motions, already King et al. (1998) pioneeristically showed that the WFPC2 on board of HST allows astrometric position measurements with a precision of the order of a few milliarcseconds (mas) on a single image. With WSO-UV FCU imagers we expect to reach accuracies of $<1 \mathrm{mas} /$ frame in the UVO channel (200-700 nm). This accuracy, taking into account the possibility of using the archive HST data, i.e. the fact that we have a very long temporal baseline (20-30 years), allows to obtain relative proper motions measurements with accuracies of $<10$ microarcsec/yr. With the high accuracy proper motion and radial velocity measurement, the distance uncertainty mainly depends on the sampling error, which goes with the $(2 \mathrm{n})^{1 / 2}$, where $\mathrm{n}$ is the number of measured stars (typically a few thousands). Using background nucleated galaxies (or, even better, background point like sources like QSO) it is also possible to have absolute proper mo- 
tions with an accuracy which is mainly related to the ability to measure the position of the reference galaxies. This accuracy can be estimated to be of the order of a 30 microarcsec (better in the case that the reference objects are background QSOs). With these absolute proper motions it is possible to:

1. use the 3-D kinematics of GCs as a probe of the Galactic potential;

2. use the 3-D kinematics, coupled with information on the GC ages and metal content, to detect Galactic streams that are important information to understand the role of the accretion from small size satellites in the assembly of our Galaxy.

\section{Young planetary disks and extrasolar planetary atmospheres}

T Tauri stars (TTSs), solar-like pre-main sequence stars, are unique to study the environment (radiation, high energy particles, dynamical processes) in which planetary systems, like ours, grow. Notice that recent theories propose that the inner, Earth-like, planets begin to build-up some $10^{6} \mathrm{yr}$ after the star begin to form and, at this stage, the accretion-based engine is still operating. The radiation produced by the engine ought to have an important effect on the inner disk evolution and the evaporation of the primary atmospheres of the planets-embryos through photoionization and photochemical reactions (Watson et al 1981, Lecavelier des Etangs et al 2004). Thus, UV spectroscopy will allow the study of the interactions between the stellar UV field and the young planetary disks and detect the molecular component in very diffuse disks (Jolly et al 1998). Very recent chemical models are showing that the penetration of UV photons coming from the central engine in a dusty disk could produce an important change in the chemical composition of the gas allowing the growth of large organic molecules. In this context, UV photons at $\lambda>1500 \AA$ photodissociating organic molecules could play a key role in the chemistry of the inner regions of the proto-planetary disk, while those photodissociating $\mathrm{H}_{2}$ and $\mathrm{CO}$ would control the chemistry of the external layers of the disk directly exposed to the radiation from the star. The radiation field can produce a rich photochemistry on timescales shorter than the dynamical evolution time scales, leading to the formation of large carbon-rich molecules such as $\mathrm{C}_{n} \mathrm{H}_{2}, \mathrm{HC}_{(2 n+1)} \mathrm{N}$, and $\mathrm{C}_{n}$. Reactions between these species and $\mathrm{H}$ and $\mathrm{H}_{2}$ may maintain their high abundances in spite of the strong radiation field emerging from the central star.

UV spectroscopy will also allow to study the properties of the atmospheres of extrasolar planets (high resolution ultraviolet spectroscopy has been the technique that has allowed detecting/studying for the first time the atmosphere of an extrasolar planet: HD 209458b). Observations of exoplanets and the detailed characterization of their atmospheres will help us understand better the physical processes at work in the building of a planet and its atmosphere, and in the further evolution of such a system. 
The WSO-UV imager will offer the chance to search for and study the ultraviolet auroral emission generated by extrasolar giant planets: i) it allows a direct detection of a planet instead of the indirect methods employed to date (radial velocity and pulsar timing); ii) the presence of an auroral signature is strictly linked to the presence of a planetary magnetic field and the evidence of this effect can not be observed with any other detection method; iii) ultraviolet auroral could contribute to characterize the near space environment around planet, so that their study could provide information about both basic atmospheric composition and the energies of the impacting particles. In general, UV wavelengths provide observational advantages compared to the optical. In fact, higher contrast ratios can be achieved in UV and the UV diffraction limit allows planets to be detected at smaller angular separations to their host stars.

In the coming decade, several ground and space-based observing programs will lead to the discovery of an extremely large number of exoplanets, in particular, near-future space missions including Corot, Kepler or GAIA will discover large numbers of exoplanets transiting their parent stars. To acquire a revealing picture of these new worlds, we need to characterize the planetary atmospheres of a large sample of these exoplanets. The observation of UV and optical absorptions occurring when an exoplanet transits its parent star are a very powerful diagnostic technique because of the strong absorption of stellar UV photons by the ozone molecule in the planetary atmosphere (see Ehenreich et al 2005).

In this manner, we expect to be able to address some important questions as: how do properties such as effective temperature, stellar type, high-energy particle environment, and metallicity of the central star alter the evolution of its planetary system? What effects do a planet's orbital parameters (orbital distance and eccentricity) have on its size, mass and potential migration during the formation process?(see Gómez de Castro et al 2006 for more details).

\section{Conclusions}

The scientific plans for WSO-UV are very ambitious, and span all of the astronomical research branches. WSO-UV will be operating in the second decade of this century, and it will be a fundamental tool for the development of astronomical knowledge, fully integrated with the many other space and ground-based observatories (including the new generation ELT telescopes) operating in the same temporal interval. The space missions operating in the WSO-UV era will provide observational data both at shorter wavelengths (e.g. Symbol-X, possibly the extended XMM and Chandra missions, etc.) and longer wavelengths (e.g. GAIA, JWST, Herschel etc.) than those covered by an UV optimized mission like WSO-UV. WSO-UV observations are a necessary, fundamental complement of the data set collected by presently operating and planned space and ground based observatories. 


\subsection{Acknowledgments}

Authors are grateful to the members of the Network for Ultraviolet Astronomy (NUVA) and to the other WSO-UV team members. AIGdC acknowledges the support by the Ministry of Science and Education through grant ESP200627265-E. IPa and GPi thank colleagues of the Italian WSO-UV Science Team for their valuable contribution, in a special way M.Nonino, and S.Marchi. The participation in the WSO-UV project in Italy is funded by Italian Space Agency under contract ASI/INAF No. I/085/06/0.

\section{References}

1. Dickinson, M., Giavalisco, M., and the GOODS Team, The Great Observatories Origins Deep Survey in The Mass of Galaxies at Low and High Redshift, eds. R. Bender \& A. Renzini, p. 324, 2003

2. Ehrenreich, D., 2005, PhD Thesis, Univ. de Paris VI.

3. Gómez de Castro, A.I., Verdugo, E. Hubble Space Telescope STIS Spectrum of RW Aurigae A: Evidence for an Ionized Beltlike Structure and Mass Ejection in Timescales of a Few Hours Astrophysical Journal, 597, 443, 2003

4. Gómez de Castro, A.I., Lecavelier des Etangs A., d'Avillez, M., Linsky, J.L., Cernicharo, J. UV capabilities to probe star formation: from the ISM to planets Astrophysics and Space Science, 303, 1, 2006

5. Gómez de Castro, A.I., Wamsteker, W., eds. Fundamental Problems in Astrophysics: requirements for future UV observatories Astrophysics and Space Science, 303, Vol 1-4, 2006

6. Katz, N., Weinberg, D.H., Hernquist, L., Miralda-Escudé Damped Ly $\alpha$ and Ly $\alpha$ absorbers in the cold dark matter model Astrophysical Journal, 457, L57, 1996

7. Lecavelier des Etangs, A., Vidal-Madjar, A., McConnell, J. C., Hébrard, G., Atmospheric escape from hot Jupiters Astronomy and Astrophysics, 418, L1, 2004

8. Reimers, D., Fechner, C., Hagen, H.-J., Jakobsen, P., Tytler, D., Kirkman, D. Intergalactic HeII absorption towards QSO 1157+3143 Astronomy and Astrophysics, 442, 63, 2005

9. Watson, A. J., Donahue, T. M., Walker, J. C. G., The dynamics of a rapidly escaping atmosphere - Applications to the evolution of earth and Venus Icarus, 48, 150, 1981. 